\title{
Evaluation of multi-starter S. cerevisiae/ D. bruxellensis cultures for mimicking and accelerating transformations occurring during barrel ageing of beer
}

\author{
Eduardo Coelho*, Mário Azevedo, José A. Teixeira, Teresa Tavares, José M. Oliveira, \\ Lucília Domingues*
}

CEB - Centre of Biological Engineering, University of Minho, 4710-057 Braga, Portugal

\section{A R T I C L E I N F O}

\section{Keywords:}

Beer ageing

Multi-starters

Volatile compounds

Metabolomics

Dekkera bruxellensis

\begin{abstract}
A B S T R A C T
During beer ageing, endogenous barrel microbes grow spontaneously and transform wort/beer composition, being Dekkera bruxellensis and Saccharomyces cerevisiae among the main contributors to the chemical and sensory profile of aged beer. This work aims at the application of multi-starter cultures to mimic and accelerate biological modifications occurring during barrel ageing of beer, in controlled fermentation processes. Co-cultures of D. bruxellensis/S. cerevisiae were conducted under conditions commonly found in barrel aged beer production: different pitching rates, high glucose concentration and presence of ethanol and wood extracts. Selective pressures and competition between yeasts influenced microbial growth and metabolite production, namely ethanol, acetic acid and target volatile compounds (esters, alcohols, terpenols, volatile acids and volatile phenols). Metabolic profiles of co-cultures combined traits of both species, and differed from those of pure cultures. Lastly, multi-starters were successfully applied in combination with wood in a controlled and accelerated fermentation process for mimicking barrel ageing transformations.
\end{abstract}

\section{Introduction}

With the increasing innovation and interest on craft and specialty beers, there is the constant search for alternative processes, microorganisms and raw materials, leading to an ever increasing multitude of alternative products. One example of specialty products are barrel aged beers, produced either by fermentation of wort in wood barrels or by storage of previously fermented beer in wood casks. During contact with wood several phenomena occur: wood compounds are extracted by the hydroalcoholic matrix (additive ageing), beverage compounds are adsorbed by wood (subtractive ageing), oxidative and polymerization reactions (chemical ageing) and, more importantly, a secondary fermentation takes part (biological ageing), transforming chemical and sensorial characteristics of the product (Coelho, Magalhães, et al., 2019). In most barrel aged beers, this secondary fermentation is the result of the spontaneous growth of microbial population, mostly endogenous to the barrel or populating the brewery atmosphere (Spitaels et al., 2014). Previous works have identified the more prominent microorganisms in the biological ageing of some beers, highlighting bacteria such as Enterobacteriaceae sp., Lactobacillus sp. and Pediococcus sp. as well as yeasts such as Saccharomyces sp., Debaryomyces sp. and
Dekkera sp. (Snauwaert et al., 2016; Spitaels et al., 2014). Among these, Dekkera sp. is considered the main contributor to aroma characteristics of barrel aged products (Colomer, Funch, \& Forster, 2019). Despite being commonly regarded as a spoilage microorganism in wine, this yeast is gaining increasing importance in the brewing and bioethanol industries due to its unique metabolic features, such as the bioconversion of hydroxycinnamic acids to volatile phenols, the Custer effect, and characteristic esterase and $\beta$-glucosidase activity (Kuo et al., 2018; Schifferdecker, Dashko, Ishchuk, \& Piškur, 2014; Smith \& Divol, 2016; Steensels et al., 2015).

Traditional barrel ageing has some disadvantages. It is usually a costly process, consuming time and space, and commonly poorly controlled, often with unpredictable end products. Several recent works have been performed to develop fermentative processes aiming to replicate biological transformations associated with barrel ageing, in a more controlled and efficient manner. Most of them have explored the application of alternative non-Saccharomyces species for the souring and flavoring of beer by sequential fermentation, being the end products profoundly affected by yeast metabolomics (Holt, Mukherjee, Lievens, Verstrepen, \& Thevelein, 2018; Osburn et al., 2018). Another alternative, acknowledged in wine production, is the simultaneous

\footnotetext{
* Corresponding authors.

E-mail addresses: e.coelho@ceb.uminho.pt (E. Coelho), luciliad@deb.uminho.pt (L. Domingues).
} 
culture of one or more species within the same matrix, by the addition of a multi-starter culture. This can be advantageous, as the interaction between both species can lead to distinct metabolomic profiles, contrasting with those of pure cultures (Ciani \& Comitini, 2015; Comitini et al., 2011). However, despite having been hypothesized and proposed in several recent works (Holt et al., 2018; Steensels et al., 2015), the possibility of using multi-starter cultures, involving Dekkera sp. and Saccharomyces sp., for biological ageing of beer still remains to be tested. This hypothesis is studied in this work by evaluating the growth and metabolomics of $S$. cerevisiae and D. bruxellensis co-cultures under selective pressures commonly found in barrel aged beer production. The application of $D$. bruxellensis/S. cerevisiae multi-starter cultures for the fermentation of beer in combination with the application of wood, is proposed in this work as an accelerated and controlled approach for mimicking and furthermore controlling the transformations typically occurring in barrel ageing.

\section{Materials and methods}

\subsection{Chemicals, materials and strains}

Calibration of GC-MS was performed resorting to the following pure standards. From Aldrich: ethyl butyrate (99\%), isoamyl acetate ( $\geq 99 \%$ ), ethyl hexanoate ( $\geq 99 \%$ ), ethyl lactate (98\%), ethyl octanoate ( $\geq 99 \%$ ), ethyl decanoate ( $\geq 99 \%$ ), diethyl succinate (99\%), ethyl hydrocinnamate $(99 \%)$, diethyl malate $(\geq 97 \%)$, linalool $(97 \%)$, $\beta$-citronellol (95\%), geraniol (98\%), isovaleric acid (99\%), octanoic acid ( $\geq 99.5 \%)$, 5-methylfurfural (99\%), cis-oak lactone ( $\geq 98.0 \%$ ), transoak lactone $(\geq 98.0 \%)$, 4-methylguaiacol ( $\geq 98.0 \%)$, eugenol $(99 \%)$, 2,6-dimetoxyphenol (99\%), 4-ethylphenol (99\%), syringaldehyde (98\%), tyrosol (98\%) and methionol (98\%). From Fluka: isobutyl acetate $(99 \%)$, ethyl phenylacetate $(\geq 99 \%), 2$-phenylethyl acetate ( $\geq 99 \%), \quad$ 2-methyl-1-propanol $\quad(\geq 99.9 \%), \quad$ 2-methyl-1-butanol ( $\geq 98 \%$ ), 3-methyl-1-butanol ( $\geq 99.8 \%$ ), furfuryl alcohol (98\%), 2phenylethanol ( $\geq 99 \%)$, propanoic acid $(\geq 99.5 \%)$, isobutyric acid $(\geq 99.5 \%)$, butanoic acid ( $\geq 99.5 \%)$, hexanoic acid $(\geq 98.0 \%)$, decanoic acid ( $\geq 98.0 \%)$, phenylacetic acid (99\%), furfural (99\%), vanillin $(\geq 98.0 \%)$ and acetoin (97\%). From Alfa Aesar: 4-ethylguaiacol (98\%). Oak wood used in this work (kindly provided by Seguin Moreau) was submerged in fortified wine used in Port wine production (kindly provided by Quinta do Portal S.A.), until total sorption was reached, with sorption conditions and wood and wine composition as described previously (Coelho, Domingues, Teixeira, Oliveira, \& Tavares, 2019). For the pure and co-culture fermentations Saccharomyces cerevisiae Belgian Abbey II (strain 1762, Wyeast Laboratories Inc.) was used. Dekkera bruxellensis used in this work was directly isolated from barrel fermentation of beer wort, using a modified DBDM medium $\left(6.2 \mathrm{~g} \mathrm{~L}^{-1}\right.$ yeast extract, $48 \mathrm{~g} \mathrm{~L}^{-1}$ ethanol, $0.1 \mathrm{~g} \mathrm{~L}^{-1} p$-coumaric acid, $0.01 \mathrm{~g} \mathrm{~L}^{-1}$ cycloheximide, $0.022 \mathrm{~g} \mathrm{~L}^{-1}$ bromocresol green, $0.1 \mathrm{~g} \mathrm{~L}^{-1}$ chloramphenicol, $0.1 \mathrm{~g} \mathrm{~L}^{-1}$ tetracycline, $20 \mathrm{~g} \mathrm{~L}^{-1}$ agar, $\mathrm{pH}$ 5.4), based on the previously described (Rodrigues, Gonçalves, Pereira-da-Silva, MalfeitoFerreira, \& Loureiro, 2001). Beer samples were aseptically collected from barrels in a local craft beer producer (Fermentum Lda.) and plated in the modified DBDM medium. Colonies of $D$. bruxellensis were preliminarily detected and identified by a green halo, isolated and confirmed by sequencing of the $18 \mathrm{~S}$ ITS rDNA region, presenting $97 \%$ homology to Dekkera bruxellensis. The yeast was stored at $-80{ }^{\circ} \mathrm{C}$ in a cryovial with glycerol for permanent stock and at $-20{ }^{\circ} \mathrm{C}$ for working stocks to be used in the fermentation assays.

\subsection{Inoculum preparation}

For the fermentation assays, inocula of $S$. cerevisiae and D. bruxellensis were grown from stocks of each species stored in glycerol at $-20{ }^{\circ} \mathrm{C}$. For this purpose, shake flasks with MYPG medium $\left(3 \mathrm{~g} \mathrm{~L}^{-1}\right.$ malt extract, $3 \mathrm{~g} \mathrm{~L}^{-1}$ yeast extract, $10 \mathrm{~g} \mathrm{~L}^{-1}$ glucose and $2 \mathrm{~g} \mathrm{~L}^{-1}$ peptone) were pitched separately with each yeast and incubated at $28{ }^{\circ} \mathrm{C}$ with orbital agitation, at a rotational speed of $150 \mathrm{~min}^{-1}$, until reaching stationary phase, as determined by optical density measurement at $600 \mathrm{~nm}$.

\subsection{Fermentation assays}

Different conditions were tested in the study of the co-culture growth and metabolomics by using different culture media. All fermentations were carried out in Erlenmeyer shake flasks fitted with cotton stoppers, incubated at $28{ }^{\circ} \mathrm{C}$ with orbital agitation, at a rotational speed of $150 \mathrm{~min}^{-1}$. Fermentations were started by pitching the studied media with the desired cell concentration, attained by previous cell count in the inoculum using a Neubauer chamber and pitching at the desired dilution. Periodic sampling in aseptic conditions assessed the profiling of cell growth and metabolite production. For an easier interpretation of the inoculum concentration used, each condition was labeled as $x \mathrm{SyD}$, where $x \mathrm{~S}$ stands for $S$. cerevisiae cellular concentration of $x \times 10^{6} \mathrm{~mL}^{-1}$ and $y \mathrm{D}$ stands for $D$. bruxellensis cellular concentration of $y \times 10^{6} \mathrm{~mL}^{-1}$ in the starter inoculum.

\subsubsection{Impact of pitching rate}

Co-culture fermentations were conducted in MYPG medium using different initial cell concentrations of D. bruxellensis and S. cerevisiae. Pitching rates were tested for both yeasts in co-culture, by increasing initial cell concentrations $\left(2 \times 10^{6} \mathrm{~mL}^{-1}, 5 \times 10^{6} \mathrm{~mL}^{-1}\right.$ and $10 \times 10^{6} \mathrm{~mL}^{-1}$ ) of one yeast while maintaining constant the initial cell concentration of the other yeast $\left(1 \times 10^{6} \mathrm{~mL}^{-1}\right)$. Cell growth was accompanied for each yeast in co-culture by flow cytometry and production of primary metabolites accompanied by High Performance Liquid Chromatography (HPLC).

\subsubsection{Increased sugar concentration}

A synthetic medium was used for mimicking wort fermentations with increased concentrations of MYPG components $\left(10 \mathrm{~g} \mathrm{~L}^{-1}\right.$ yeast extract, $10 \mathrm{~g} \mathrm{~L}^{-1}$ malt extract, $8 \mathrm{~g} \mathrm{~L}^{-1}$ peptone and $140 \mathrm{~g} \mathrm{~L}^{-1}$ glucose).

\subsubsection{Impact of ethanol and wood extract concentration}

For the evaluation of the impact of ethanol and wood extracts, MYPG medium was supplemented with a hydroalcoholic oak wood extract. Hydroalcoholic wood extract was obtained by extraction of used oak wood content by contact of grounded used wood with a hydroalcoholic solution of $55 \%$ ethanol, by volume, at $20^{\circ} \mathrm{C}$, during $48 \mathrm{~h}$. The liquid fraction of the extract was sterilized by filtration at $0.22 \mu \mathrm{m}$ and diluted in MYPG medium to final ethanol concentrations, by volume, of 6\% (WE6\%), 8\% (WE8\%) and 10\% (WE10\%).

\subsubsection{Application to beer ageing}

Lastly, co-culture fermentations were carried out using real beer matrices. For mimicking barrel aged beer, a commercial lager beer was put in contact with the used oak wood at different temperatures, namely $30{ }^{\circ} \mathrm{C}$ (WB30) and $50{ }^{\circ} \mathrm{C}$ (WB50) during $48 \mathrm{~h}$ following the reported in a previous work (Coelho, Teixeira, Domingues, Tavares, \& Oliveira, 2019). Aged beer was sterilized by filtration with $0.22 \mu \mathrm{m}$ sterile filters, and pitched with each species at the desired rate.

\subsection{Determination of culture growth}

Growth of each species in the co-culture was evaluated by flow cytometry using an EC800 flow cytometer (Sony Biotechnology). Gates defining S. cerevisiae and D. bruxellensis events were previously determined by analyzing pure cultures of each yeast, combining the FS, SS and FL1 parameters, indicators of size, morphology and autofluorescence of each species. Sterile PBS was used as carrier liquid with a $40 \mu \mathrm{L} \mathrm{min}^{-1}$ flow. Analysis and quantification were performed using the equipment software. 


\subsection{Analysis of primary metabolites}

Primary metabolites (glucose, ethanol and acetic acid) were analyzed by a high performance liquid chromatography (HPLC) system composed by a Jasco PU-1580 pump, a Jasco AS-2057 autosampler, a Jasco RI-2031 Plus RI detector and a Knauer K-2501 UV detector at the wavelength of $210 \mathrm{~nm}$. $20 \mu \mathrm{L}$ of each sample were injected in an Aminex HPX-87H (BioRad) column, maintained at $60{ }^{\circ} \mathrm{C}$, using $5 \mathrm{mmol} \mathrm{L}^{-1} \mathrm{H}_{2} \mathrm{SO}_{4}$ as mobile phase. Chromatograms were analyzed using the Star-Chromatography Workstation software (version 6.9.3, Varian), and compounds were quantified against calibration curves prepared from pure standards.

\subsection{Analysis of secondary metabolites}

Volatile compounds in the samples were analyzed by gas chromatography coupled with mass spectrometry (GC-MS), following the procedure proposed by Coelho and collaborators (Coelho et al., 2020). Each $8 \mathrm{~mL}$ sample was extracted with $400 \mu \mathrm{L}$ of dichloromethane (SupraSolv for gas chromatography, Merck), after adding 4-nonanol as internal standard $(3.2 \mu \mathrm{g})$. Extractions were performed in Pyrex tubes fitted with Teflon caps, with agitation promoted by a stir bar during 15 min. Extracts were then recovered with a glass Pasteur pipette, dehydrated with anhydrous sodium sulfate and analyzed in a Varian 3800 gas chromatograph equipped with a 1079 injector and an ion-trap mass spectrometer Varian Saturn 2000. Each $1 \mu \mathrm{L}$ injection was made in splitless mode (30 s) in a Sapiens-Wax MS column (30 m $\times 0.15 \mathrm{~mm}$; $0.15 \mu \mathrm{m}$ film thickness, Teknokroma). Carrier gas was helium 49 (Praxair) at a constant flow of $1.3 \mathrm{~mL} \mathrm{~min}^{-1}$. The detector was set to electronic impact mode with an ionization energy of $70 \mathrm{eV}$, a mass acquisition range $(\mathrm{m} / \mathrm{z})$ from 35 to 260 and a $610 \mathrm{~ms}$ acquisition interval. The oven temperature was initially set to $60{ }^{\circ} \mathrm{C}$ for $2 \mathrm{~min}$ and then raised to $234{ }^{\circ} \mathrm{C}$ at a rate of $3{ }^{\circ} \mathrm{C} \mathrm{min}-1$, raised again to $260{ }^{\circ} \mathrm{C}$ at $5{ }^{\circ} \mathrm{C} \mathrm{min}{ }^{-1}$ and finally maintained at $260{ }^{\circ} \mathrm{C}$ for $10 \mathrm{~min}$. Injector temperature was set to $250{ }^{\circ} \mathrm{C}$ with a $30 \mathrm{~mL} \mathrm{~min}^{-1}$ split flow and transfer line was maintained at $250{ }^{\circ} \mathrm{C}$. Compounds were identified using MS Workstation version 6.9 (Varian) software, by comparing mass spectra and retention indices with those of pure standards and quantified as 4nonanol equivalents.

\subsection{Statistical analysis}

Statistically significant differences in concentrations of volatile compounds were evaluated by a non-parametric Kruskall-Wallis analysis, with Conover-Iman multiple pairwise comparisons, using the XLStat software (Addinsoft). For evaluation of correlations between samples and volatile compounds a multivariate approach was performed by Principal Component Analysis (PCA) using Statistica software (version 7, StatSoft Inc.)

\section{Results and discussion}

\subsection{Effect of pitching rate}

A preliminary assay evaluating the impact of pitching concentration on the growth of each yeast in co-culture was performed. Synthetic MYPG medium was pitched with different concentrations of $D$. bruxellensis and $S$. cerevisiae for the evaluation of growth and metabolite production, with results presented in Fig. 1. As seen, the favoring of each species by means of higher inoculum led to its more prominent growth in the co-culture. A difference in cellular concentration of only $1 \times 10^{6} \mathrm{~mL}^{-1}$ higher in the pitching rate was sufficient for one yeast to be dominant over the other. This is noticeable in Fig. 1a), where $D$. bruxellensis growth is clearly dominant over $S$. cerevisiae for all of the favoring inoculum concentrations of D. bruxellensis. Despite contributing for a dominant growth, increasing cellular pitching concentration above $2 \times 10^{6} \mathrm{~mL}^{-1}$, did not contribute to a significant increase of $D$. bruxellensis growth rate or maximum growth. However, the increasing concentrations of $D$. bruxellensis in the inoculum led to a proportionally lesser growth of $S$. cerevisiae in the co-culture. On the other hand, this behavior may also be seen when favoring $S$. cerevisiae in the inoculum, as visible in Fig. 1b). In this case, a higher pitching rate of $S$. cerevisiae also led to a dominance in its growth and an inhibition of $D$. bruxellensis. Increasing S. cerevisiae initial cellular concentration in the inoculum over $2 \times 10^{6} \mathrm{~mL}^{-1}$ also did not further favor $S$. cerevisiae growth, but on this hand no proportionality in D. bruxellensis growth inhibition could be observed. This phenomenon of growth dominance of one species over the other also impacts the production of primary metabolites in the co-culture. As visible in Fig. 1c) the predominance of D. bruxellensis led to a higher production of its typical metabolites, particularly acetic acid. In fact, acetic acid production was only observed in the co-cultures favoring $D$. bruxellensis growth, being absent in the co-cultures promoting $S$. cerevisiae, as visible in Fig. 1d). Maximum ethanol concentration was similar in both scenarios, reaching values of about $4 \mathrm{~g} \mathrm{~L}^{-1}$ either favoring $D$. bruxellensis or $S$. cerevisiae in the inoculum. In both conditions, when sugar depletion was complete, ethanol concentration began to decrease significantly, reaching residual values even in the $D$. bruxellensis favoring condition. Both yeast can metabolize ethanol as carbon source as part of a make-accumulateconsume mechanism, natural for both species (Rozpedowska et al., 2011). However, cell growth accompanying the depletion of ethanol was more prominent in the case of D. bruxellensis, which is better adapted to grow under this condition (Smith \& Divol, 2016). In contrast, on the $S$. cerevisiae favoring condition, no acetic acid production was observed, despite the occurrence of $D$. bruxellensis growth and the decrease in ethanol concentration. This absence of acetic acid production can be firstly due to the rapid depletion of glucose by the outcompeting $S$. cerevisiae, leaving no substrate available to $D$. bruxellensis other than ethanol. On the other hand, there is the rapid decrease of available oxygen in the fermentation medium and an anaerobic condition being imposed by the $\mathrm{CO}_{2}$ produced by $S$. cerevisiae. As described, anaerobic conditions hinder acetic acid production by D. bruxellensis (Smith \& Divol, 2018). This absence in the production of acetic acid is of particular interest when envisaging beverage production, which can be targeted or not depending on the intended product. Therefore, the presence or absence of $S$. cerevisiae in the starter culture can be a tool to manage acetic acid concentrations in the end product.

\subsection{Fermentation with high glucose concentration}

In order to evaluate the production of secondary metabolites and coculture performance with alternative conditions, a fermentation with high glucose concentration $\left(140 \mathrm{~g} \mathrm{~L}^{-1}\right)$ was performed. Based on the previous results, two different inoculum concentrations were chosen, one favoring $D$. bruxellensis and another favoring S. cerevisiae, accompanied by controls of each species grown in pure cultures. Cell growth and primary metabolite production profiles are presented in Fig. 2. In contrast with the previous results obtained in synthetic medium with low sugar concentration $\left(10 \mathrm{~g} \mathrm{~L}^{-1}\right)$, a clear dominance of $S$. cerevisiae has been observed for all the conditions in the co-cultures, as visible in Fig. 2a). The well acknowledged adaptation of $S$. cerevisiae to high sugar concentrations and its higher growth rates make it a dominant species over D. bruxellensis when such condition is imposed (Abbott, Hynes, \& Ingledew, 2005; Blomqvist, 2011). Aguilar-Uscanga and collaborators have previously demonstrated the inhibitory effect of this selective pressure on $D$. bruxellensis performance, reporting decreases in growth rate, substrate consumption and metabolite production with increasing glucose concentrations, being $165 \mathrm{~g} \mathrm{~L}^{-1}$ the borderline from which these losses become more significant (Aguilar-Uscanga et al., 2011). Therefore, even with inoculum favoring $D$. bruxellensis, S. cerevisiae was dominant in both co-cultures contrasting with the previous results. In fact, $D$. bruxellensis is only able to outcompete $S$. cerevisiae when glucose 

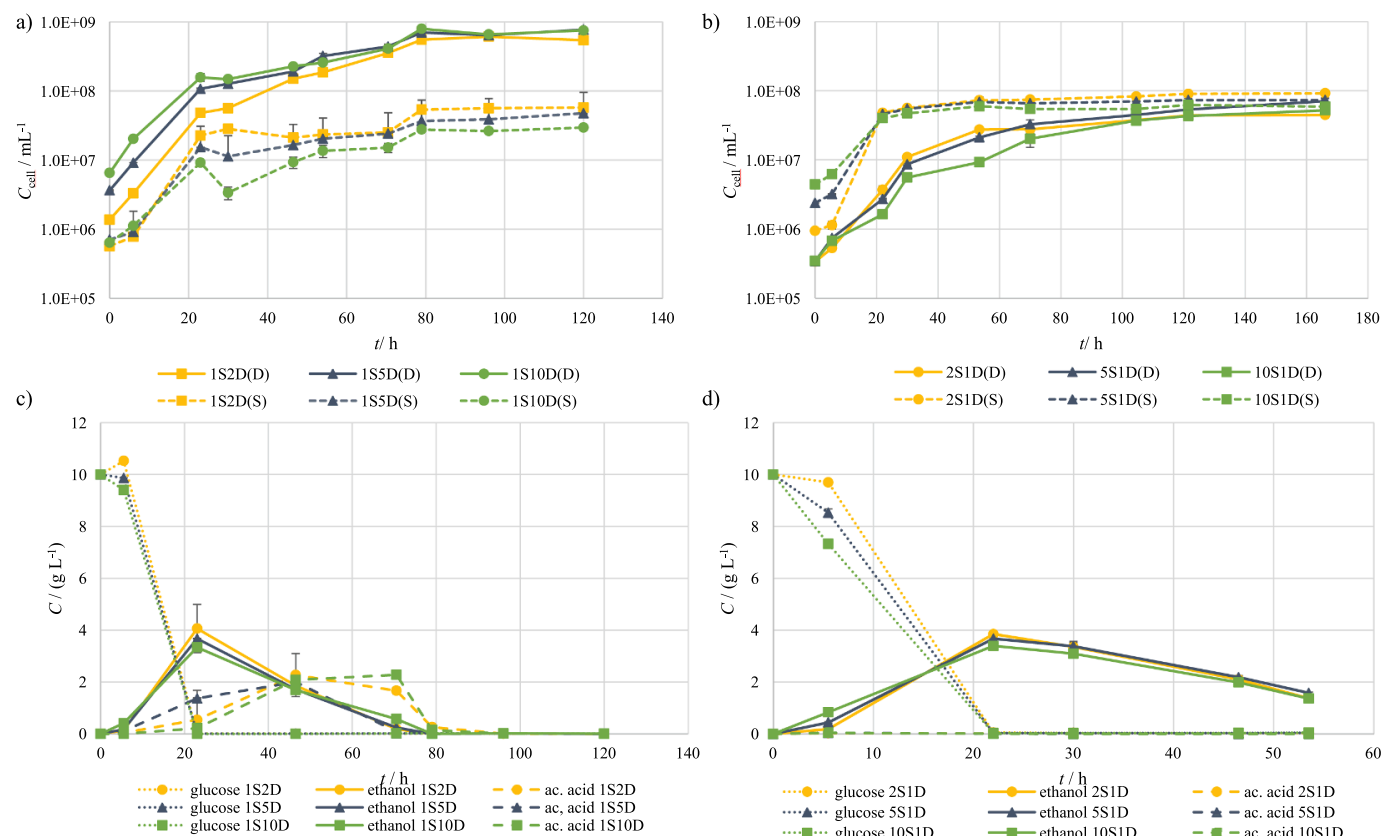

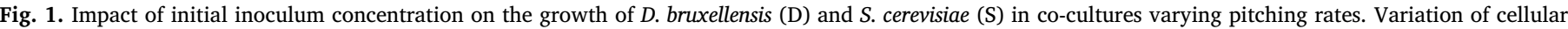

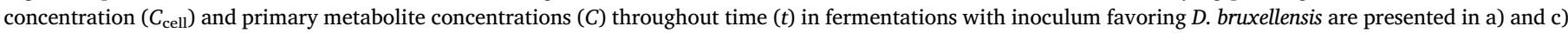

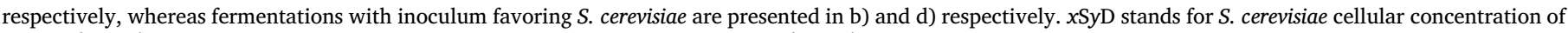

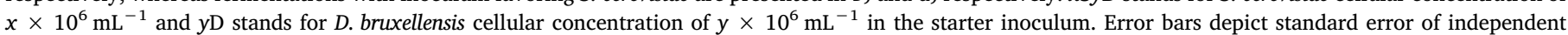
duplicates.

concentrations in the medium are low, due to different efficiencies of glucose transporters with dissimilar affinities (Blomqvist, 2011), justifying the distinct behaviors observed for low and high sugar concentrations. Despite the superiority of $S$. cerevisiae, D. bruxellensis was still able to grow in both co-cultures, but at lower levels when compared to the pure culture, due to the rapid depletion of substrate. Even with the dominance of $S$. cerevisiae, $D$. bruxellensis still produced considerable amounts of acetic acid, as visible in Fig. 2b), being at lower concentrations in the co-cultures when compared with the pure culture. Again, maximum ethanol production was similar for $S$. cerevisiae and D. bruxellensis pure cultures, but it was reached at different times due to the differences in the specific growth and production rates of the two species.

Digging deeper into species metabolomics, in either pure or coculture conditions, secondary metabolite production was characterized, and is presented in Table 1. Also, to better describe the correlations in production of secondary metabolites, a PCA was performed and a scatterplot was outlined, which is presented in Fig. 3. Production of metabolites was found to be highly species dependent, either in terms of the compounds formed or their levels of production. For instance, isobutyl acetate was only found in cultures with $D$. bruxellensis. Production of this ester was even higher in co-cultures of D. bruxellensis and $S$. cerevisiae, when compared with pure culture of D. bruxellensis, contrasting with the lower growth and primary metabolite production observed. Moreover, increasing D. bruxellensis concentration in the coculture inoculum led to an increase in isobutyl acetate production when compared with the co-culture using lower concentrations of D. bruxellensis. This may indicate a synergy between the two yeasts in the production of this ester. Tashiro and collaborators have proposed a twoway pathway for the production of isobutyl acetate involving glucose with 2-methyl-1-propanol and/or acetate as intermediaries (Tashiro, Desai, \& Atsumi, 2015). Taking into account that $S$. cerevisiae produced high concentrations of 2-methyl-1-propanol, which $D$. bruxellensis produces at lower levels, and that $D$. bruxellensis produces high concentrations of acetate, the synergy between these two yeasts can be the driver of the higher isobutyl acetate production observed, with one species producing a substrate used by the other. Ethyl hexanoate and ethyl octanoate also showed higher concentrations in the co-culture than in pure cultures of each yeast. These esters can be formed by reaction of an alcohol and a carboxylic acid group, either driven by enzymatic activity or from chemical reaction at low pH (Sumby, Grbin, \& Jiranek, 2010). One of the features of D. bruxellensis, which highly imparts beverage composition, is its characteristic esterase activity that can lead to both hydrolysis and synthesis of esters. Therefore, D. bruxellensis can be responsible for esterification of middle and long chain fatty acids with high specificity towards the formation of ethyl esters (Colomer et al., 2019; Spaepen \& Verachtert, 1982). On the other hand, these esters can derive from chemical reaction of the corresponding fatty acids with ethanol. In fact, concentration of several volatile fatty acids was higher in $D$. bruxellensis pure cultures when compared with S. cerevisiae. Synthesis of fatty acids is acknowledged as another typical D. bruxellensis trait (Steensels et al., 2015). These compounds are generally a product of sugar and aminoacid catabolism, via the Ehrlich pathway (Hazelwood, Daran, Van Maris, Pronk, \& Dickinson, 2008). In contrast with $S$. cerevisiae, $D$. bruxellensis typically diverts aminoacid conversion towards production of fusel acids instead of producing fusel alcohols, due its increased alcohol and aldehyde dehydrogenase activity (Piškur et al., 2012). As expected, D. bruxellensis fermentation in pure culture produced high concentrations of volatile fatty acids. However, production of fatty acids in co-culture fermentations occurred at levels close to those seen in the $S$. cerevisiae pure cultures and significantly lower to those observed for $D$. bruxellensis pure cultures. This can be a direct consequence of the competitive advantage of $S$. cerevisiae for sugar and aminoacid metabolism. As $S$. cerevisiae has an advantage, conversion of sugars and aminoacids via the Ehrlich pathway will be more directed towards the production of fusel alcohols instead of fusel acids (Hazelwood et al., 2008; Yu, Pratomo Juwono, Foo, Leong, \& Chang, 2016). In agreement, concentrations of alcohols were higher in $S$. cerevisiae pure culture, when compared with $D$. bruxellensis, attaining similar concentration values in the co-culture fermentations. This is a clear advantage of co-fermentation of D. bruxellensis with $S$. cerevisiae, considering that fatty acids impart beverages with unwanted sensory 


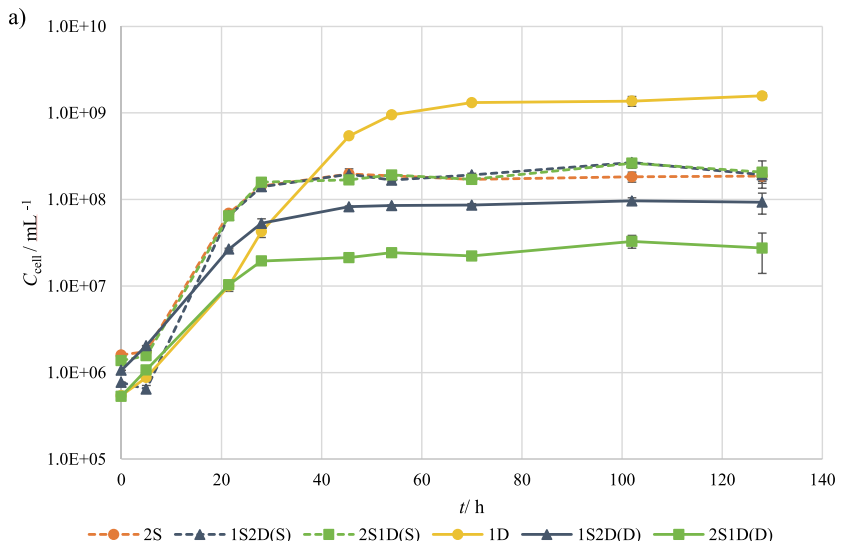

b)

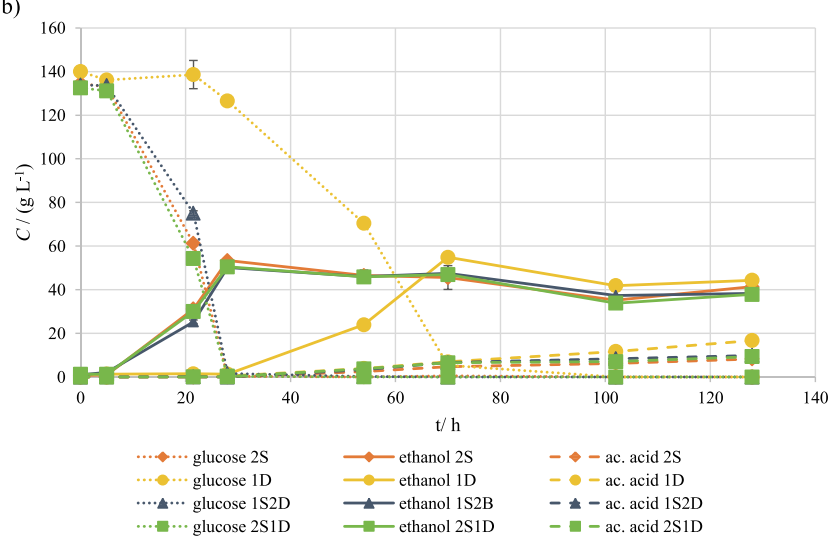

Fig. 2. Fermentation profiles depicting a) Cellular concentration $\left(C_{\text {cell }}\right)$ of $S$. cerevisiae (S) and D. bruxellensis (D) and b) primary metabolite concentrations $(C)$ throughout time $(t)$, in fermentations using pure cultures (2S and 2D) and co-cultures favoring S. cerevisiae (2S1D) or D. bruxellensis (1S2D) in the multistarter. $x$ SyD stands for $S$. cerevisiae cellular concentration of $x \times 10^{6} \mathrm{~mL}^{-1}$ and $y \mathrm{D}$ stands for $D$. bruxellensis cellular concentration of $y \times 10^{6} \mathrm{~mL}^{-1}$ in the starter inoculum. Error bars depict standard error of independent duplicates.

features and are commonly regarded as spoilage compounds. Typical volatile phenols, 4-ethylphenol and 4-ethylguaiacol were exclusively found associated with $D$. bruxellensis growth, widely acknowledged for this yeast (Suárez, Suárez-Lepe, Morata, \& Calderón, 2007). Co-culture of $D$. bruxellensis with $S$. cerevisiae led to a decrease in ethylphenol production, again due to the hindered $D$. bruxellensis growth by competition with $S$. cerevisiae for the substrate. Despite being considered spoilage compounds in wine, volatile phenols produced by D. bruxellensis are a desirable trait in barrel aged beers being characteristic in the aroma of such products. Also advantageous to beer aroma, monoterpenic alcohols were produced in higher concentrations by D. bruxellensis when compared with $S$. cerevisiae. De novo production of terpenoids is an acknowledged trait of yeast, already documented for Saccharomyces sp. (Carrau et al., 2005) and multiple other non-Saccharomyces sp. yeasts (Rossouw \& Bauer, 2016) with the exception of D. bruxellensis for which it is still not well documented. Previous works have provided clues about the capacity of Dekkera sp. to produce terpenes, hinting production of terpenoids possibly linked to $\beta$-farnesene (Joseph, Albino, Ebeler, \& Bisson, 2015; Joseph, Gorton, Ebeler, \& Bisson, 2013). Nevertheless, terpenes produced by $D$. bruxellensis in this work were more similar to the ones produced by $S$. cerevisiae, however at significantly higher titers. De novo production of linalool and geraniol by $D$. bruxellensis is a novel and significant feature reported for this yeast. These monoterpenes impart beer with desirable floral aroma, being generally correlated with hops descriptors (Denby et al., 2018). Similarly to other metabolites previously discussed, geraniol and linalool concentrations where higher in pure culture of D. bruxellensis, being their concentration lower in co-cultures due to S. cerevisiae competition. Analyzing correlations among the produced metabolites presented in the scatterplot in Fig. 3a), three clusters can be distinguished from the two extracted components highlighting the compounds that better correlate with each species. As previously referred, several metabolites were closely related to $D$. bruxellensis pure culture, namely the volatile phenols 4-ethylphenol and 4-ethylguaiacol, the monoterpenes linalool and geraniol and the acids isovaleric and phenylacetic. On the $S$. cerevisiae cluster, the esters ethyl butyrate and 2phenylethyl acetate and acetoin arise with closer relation to this species. More importantly, co-cultures of S. cerevisiae and D. bruxellensis clearly show the combination of metabolite features of both yeasts, correlating with typical and common metabolites of each species either by the first and/or the second component.

\subsection{Fermentation with addition of wood extract}

In order to mimic fermentation of barrel aged beer, a preliminary assay was conducted using synthetic MYPG medium with addition of oak wood extract. To better assess the effect of ethanol in combination with wood composition, generally extracted from oak barrels, co-cultures were conducted by addition of oak extracts at different ethanol percentages. Growth of each species in the co-culture is presented in Fig. 4a). As visible, yeast growth was affected by increasing concentrations of alcoholic oak extract in the medium, possibly due to a synergistic inhibition effect of both ethanol and compounds extracted from oak. Inhibitory effect of ethanol on yeast growth is well known both for S. cerevisiae and for D. bruxellensis. As described by Bassi and collaborators, an increase of ethanol concentration from $9 \%$ to $10 \%$ causes a significant decrease both in S. cerevisiae and D. bruxellensis growth, being this inhibition complete when increasing ethanol percentage up to $11 \%$ (Bassi, Silva, Reis, \& Ceccato-Antonini, 2013). Besides ethanol, increasing oak extract concentration also increases content of typical wood inhibitors in the fermentation medium. Oak extracts have significant amounts of phenolic compounds such as furans and phenolic acids (Coelho, Teixeira, et al., 2019). This is visible in Table 1 for the dilution corresponding to $10 \%$ ethanol, where microbial growth was absent. Thus syringaldehyde, vanillin, furfural, 5-methylfurfural and 5-hydroxymethylfurfural were initially present in the medium, all coming from the oak wood extract. Impact of these compounds on $S$. cerevisiae and D. bruxellensis metabolism is well known in the field of bioethanol production; for instance furan compounds are strong inhibitors of $S$. cerevisiae and $D$. bruxellensis growth in pretreated lignocellulosic medium (Blomqvist et al., 2011; Kelbert et al., 2016). It is important to highlight that final concentration of the abovementioned inhibitors was practically residual in the cultures where $S$. cerevisiae and $D$. bruxellensis were able to grow. As reported, $S$. cerevisiae can detoxify the medium by converting furans to least harmful compounds, enduring considerable concentrations of these inhibitors (Cunha, Romaní, Costa, Sá-Correia, \& Domingues, 2019). On its hand, D. bruxellensis also endures low to moderate concentrations of these compounds but with its fermentative performance strongly affected (Galafassi et al., 2011).

In contrast with the previous fermentation using high sugar concentration, D. bruxellensis showed a competitive advantage over $S$. cerevisiae in the co-culture fermentations with oak extract. Ethanol and glucose concentrations initially available in the medium were key aspects for this competitive advantage. As previously mentioned, $D$. bruxellensis is able to outcompete $S$. cerevisiae only when initial glucose concentrations in the medium are low (Blomqvist, 2011). Another aspect to take into account in its competitive advantage is $D$. bruxellensis ability to grow and metabolize alternative carbon sources efficiently, for example ethanol, pentoses and even cellobiose (Blomqvist, Eberhard, Schnürer, \& Passoth, 2010). As presented in Fig. 4b), a total depletion of glucose is observed in the co-cultures for the dilutions corresponding to $6 \%$ and $8 \%$ ethanol. For the condition of $10 \%$, 


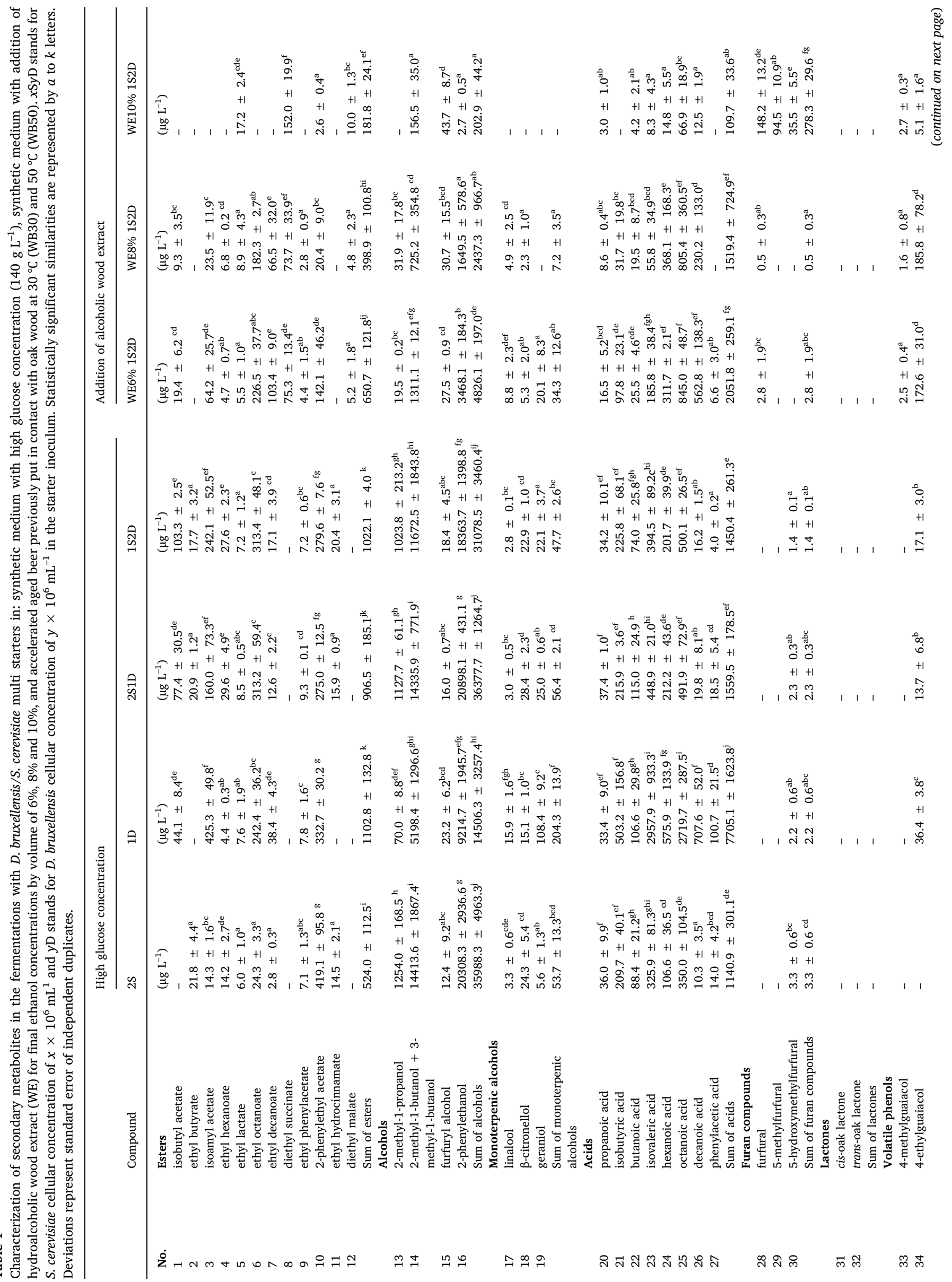




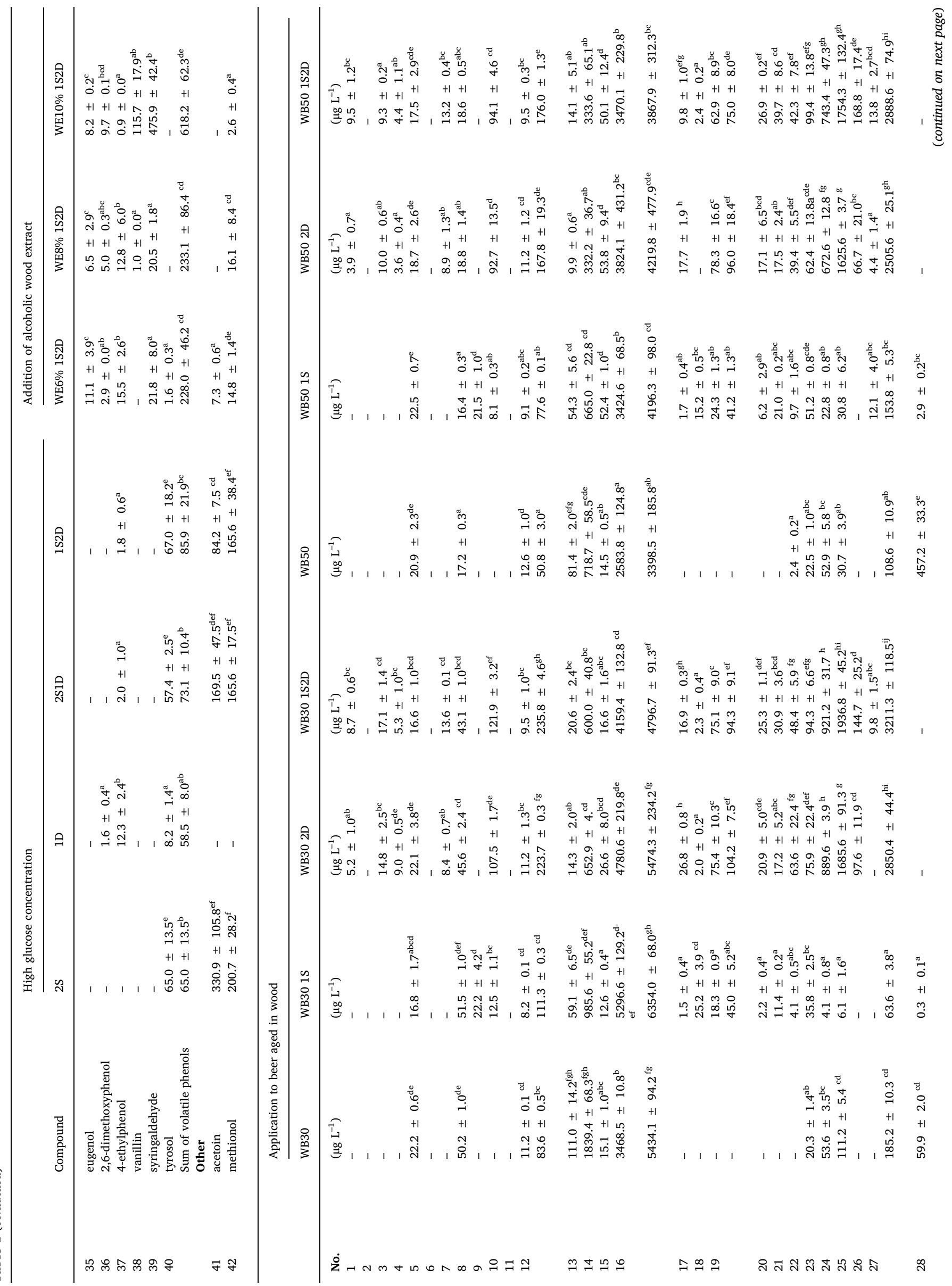




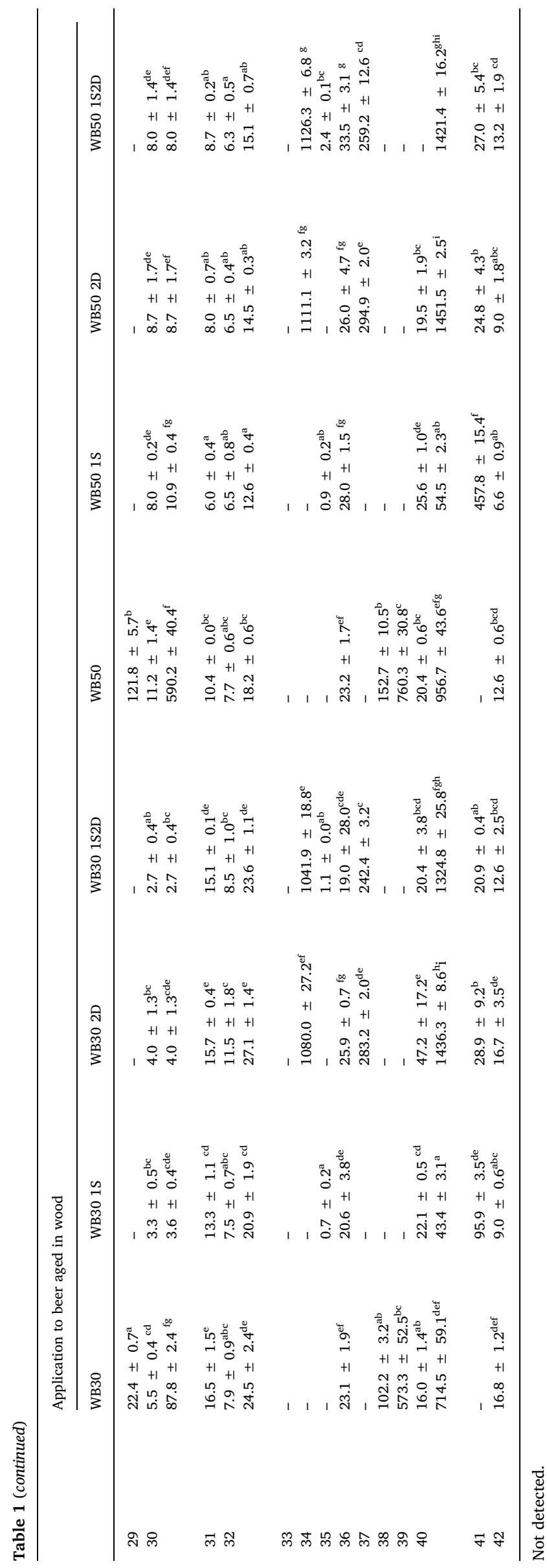

glucose, concentration did not vary due to absence of microbial growth. A slower sugar conversion and acetic acid production rates were observed in the co-cultures for the dilution of oak extract to $8 \%$ ethanol, when compared with the $6 \%$ condition, naturally linked to the increased selective pressure on the co-culture. This selective pressure affected both species equally as seen for microbial growth, which is reflected in all of the primary metabolites studied.

Focusing on secondary metabolites, several compounds, previously identified in the high sugar concentration fermentations, were produced in the fermentations with addition of oak extract. Several esters were found, with particular emphasis on ethyl esters previously associated to $D$. bruxellensis, namely ethyl octanoate, ethyl decanoate and 2phenylethyl acetate. Isobutyl acetate, previously identified as deriving from a potential synergy between $S$. cerevisiae and $D$. bruxellensis, also appeared in fermentations where microbial growth occurred. Considerable amounts of fatty acids were also found in fermentations with oak extract, along with rather low concentrations of alcohols, reflecting the more pronounced growth of $D$. bruxellensis in the fermentations with oak extract. As visible in Table 1, concentration of furan compounds was almost null in the fermentations where microbial growth was observed, an indicator of their detoxification. Also, a more pronounced formation of ethylphenols was observed, a direct result of a higher concentration of hydroxycinnamic acids in the medium, which typically derives from the wood extract. Several other compounds were found, which derived from the oak wood extract and are not metabolites of neither of the species. Such is the case of diethyl succinate, ethyl lactate and diethyl malate, which were adsorbed by wood in its prior contact with wine and posteriorly extracted from wood, coherent with the reported in previous works (Coelho, Domingues, et al., 2019). Among these, diethyl succinate and diethyl malate concentration decreased significantly in the cultures where microbial growth was observed, potentially due to $D$. bruxellensis esterase activity. Scatterplot presented in Fig. 3b), demonstrates the correlation of samples corresponding to $6 \%$ and $8 \%$ dilutions of oak extract with the compounds derived from yeast metabolism. Higher correlations were found for coculture fermentations of diluted oak extract to $6 \%$ due to the increased microbial growth and metabolism. Nevertheless, fermentations of oak extract corresponding to $8 \%$ ethanol appeared closely in the first component. On the other hand, sample corresponding to $10 \%$ ethanol appeared in the cluster correlating closer to wood compounds from oak extract, demonstrating the absence of microbial activity.

\subsection{Application to beer ageing}

Lastly, to further validate the results, single species and co-culture fermentations were conducted in real beer matrixes. For this purpose, beer was previously put in contact with used oak wood from wine ageing to induce oak wood characteristics by an accelerated approach. Two different temperatures were used for contact between oak wood and beer, in order to obtain different beer compositions, following previous findings (Coelho, Teixeira, et al., 2019). Thus, beer was previously put in contact with used oak wood chips at $30{ }^{\circ} \mathrm{C}$ (WB30) and $50{ }^{\circ} \mathrm{C}$ (WB50) during $48 \mathrm{~h}$ to obtain dissimilar accelerated aged beers with different concentrations of wood extractives. Due to the higher contact temperature, WB50 was characterized by a higher content in wood extractives, namely furan compounds and aldehydes, as presented in Table 1. Moreover, several other phenolic compounds can be extracted from wood, as for example phenolic acids such as syringic acid, ellagic acid and several other polyphenols (Coelho, Teixeira, et al., 2019), which can impair yeast growth and metabolism. Nevertheless, as presented in Fig. 5a), both yeasts were able to grow in both aged beers, even with the low sugar content available and the presence of ethanol and inhibitors. Both yeasts withstood higher concentrations of inhibitory compounds (furfural, 5-methylfurfural, vanillin and syringaldehyde) when compared with fermentations in synthetic medium with addition of oak extracts, presented in Table 1 . However, ethanol 
a)

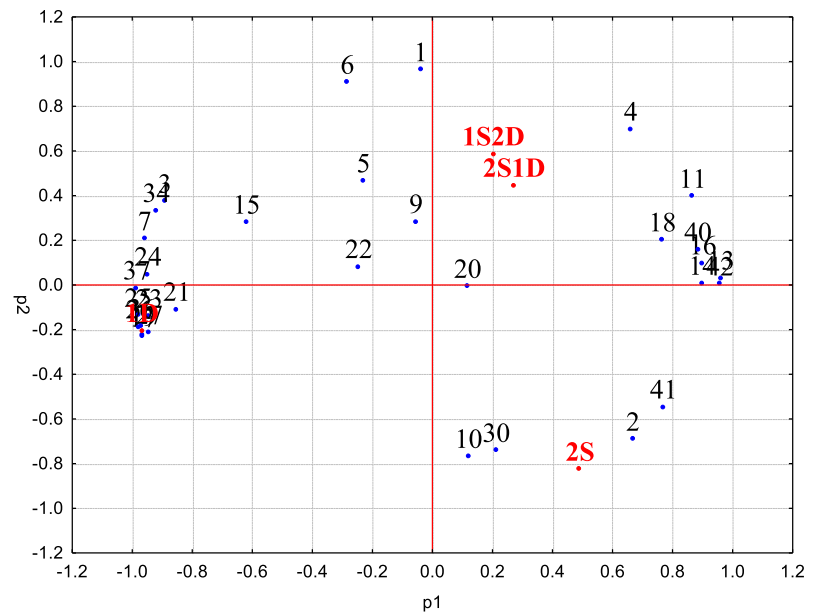

b)

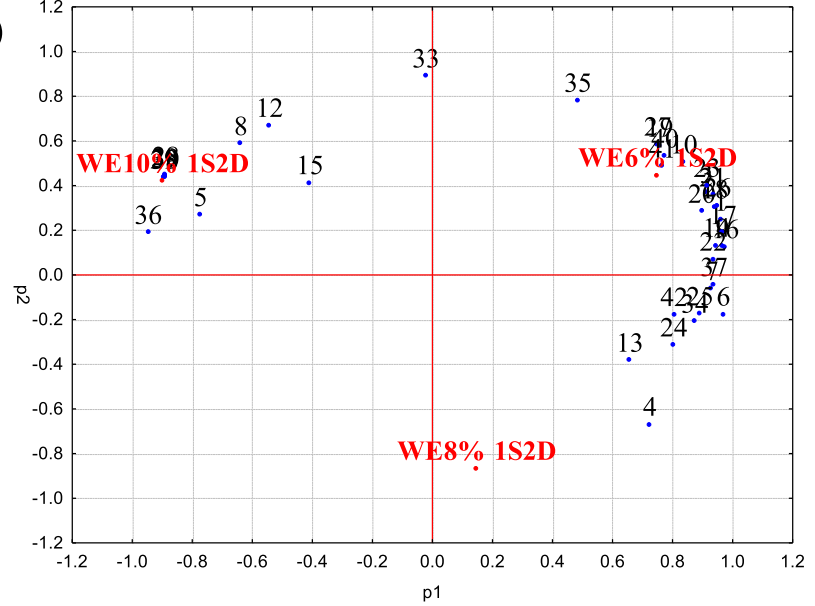

c)

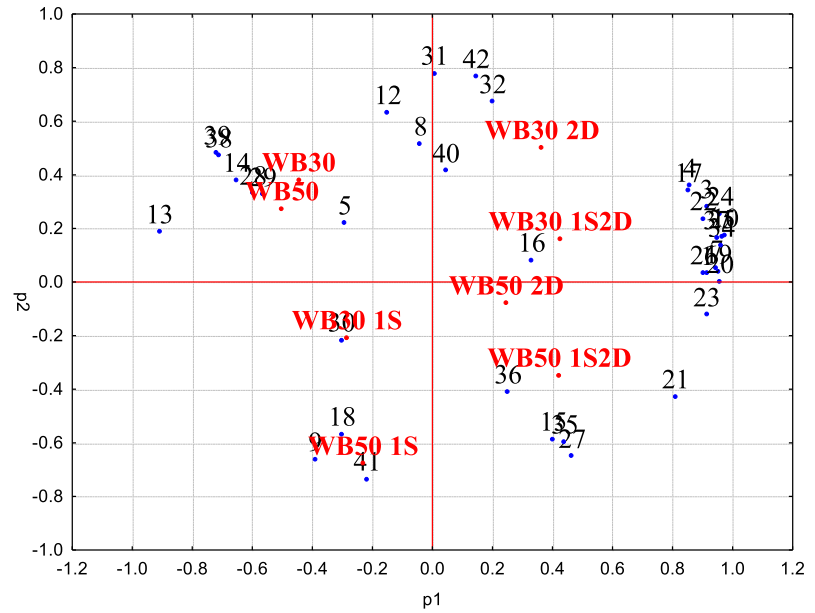

Fig. 3. Scatterplots correlating metabolites and pure and/or co-cultures of $S$. cerevisiae and D. bruxellensis for fermentation of a) synthetic medium with high glucose concentrations, b) synthetic medium with addition of oak extract at ethanol concentrations by volume of 6\% (WE6\%), 8\% (WE8\%) and 10\% (WE10\%) and c) biological ageing of beer after contact with reused oak wood at $30{ }^{\circ} \mathrm{C}$ (WB30) and $50{ }^{\circ} \mathrm{C}$ (WB50). Numbers in the scatterplot refer to the volatile compounds presented in Table 1. xSyD stands for S. cerevisiae initial cellular concentration of $x \times 10^{6} \mathrm{~mL}^{-1}$ and $y \mathrm{D}$ stands for $D$. bruxellensis initial cellular concentration of $y \times 10^{6} \mathrm{~mL}^{-1}$ in the starter inoculum.

concentrations in the aged beers were significantly lower, which led to lesser inhibitory stress and provided adequate conditions for growth. Competitive advantage of one species over another was not evident, as both species presented similar microbial growth in co-culture and single culture fermentations. As presented in Fig. 5b), acetic acid production

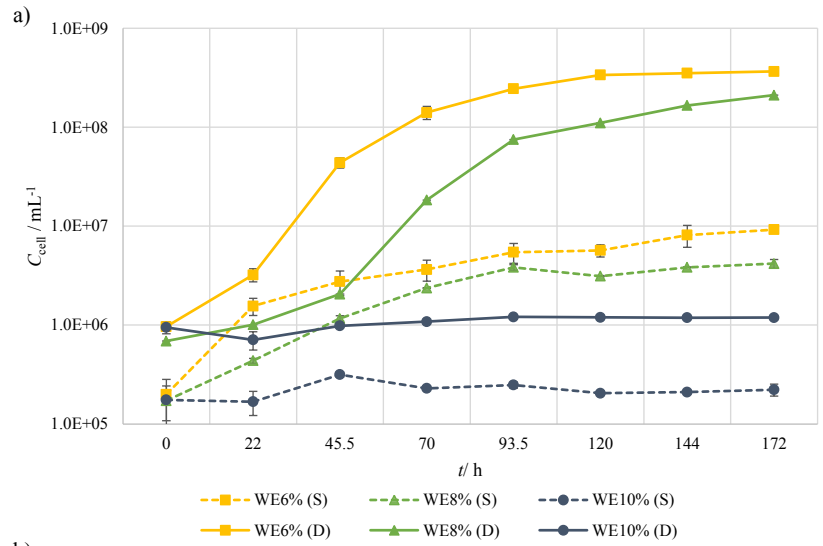

b)

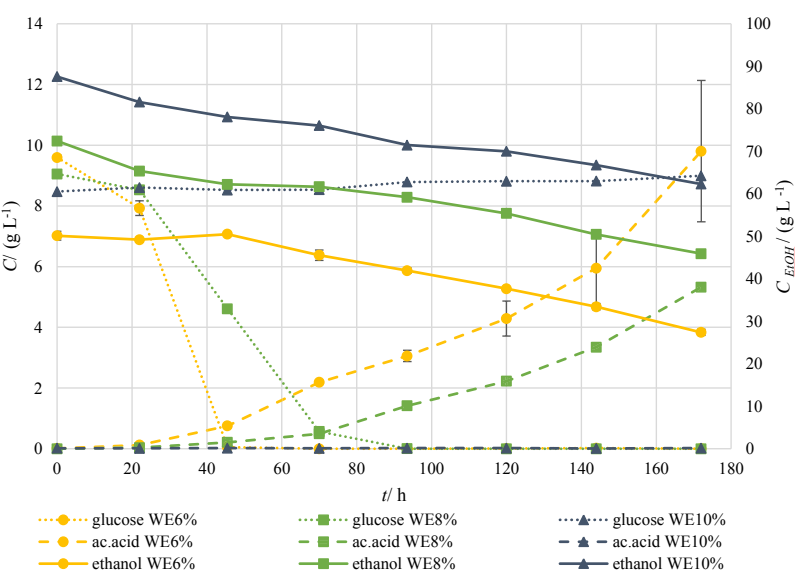

Fig. 4. Fermentation profiles of synthetic medium with addition of hydroalcoholic wood extracts depicting a) Cellular concentration ( $\left.C_{\text {cell }}\right)$ of $S$. cerevisiae (S) and D. bruxellensis (D) and b) primary metabolite concentration $(C)$ throughout time $(t)$, in fermentations pitched with cellular concentration of $1 \times 10^{6} \mathrm{~mL}^{-1}$ of $S$. cerevisiae and $2 \times 10^{6} \mathrm{~mL}^{-1} \mathrm{D}$. bruxellensis in the starter inoculum. Ethanol concentration $\left(C_{\mathrm{EtOH}}\right)$ is plotted in the secondary axis, whereas the remaining are plotted in the main axis. Error bars depict standard error of independent duplicates.

was only observed in pure and co-cultures including D. bruxellensis and absent in pure cultures of $S$. cerevisiae.

Focusing secondary metabolites, formation and depletion of several compounds previously identified in the assays with synthetic media occurred in the fermentations of the accelerated aged beers either with pure or co-cultures. As visible in Table 1, several inhibitory compounds found in the accelerated aged beers, namely furfural, 5-methylfurfural, vanillin and syringaldehyde, were metabolized, corroborating the results obtained in the fermentations with wood extract. This bioconversion, performed by both yeasts, can be disadvantageous when envisaging incorporation of wood compounds in beer. These compounds are often associated with sweet desirable aromas, which are lost due to microbial growth and metabolism. The only compounds related with oak wood aroma that were maintained after fermentation of accelerated aged beers were cis- and trans-oak lactones, typically associated with coconut aroma descriptors, and 2,6-dimethoxyphenol. Also, concentrations of ethyl lactate, diethyl succinate and diethyl malate were maintained in the same range. These compounds were adsorbed from wine by wood in its previous contact and migrated to beer by reutilization of oak wood (Coelho, Teixeira, et al., 2019). Therefore, microbial activity on accelerated aged beers did not affect significantly the occurrence of typical wine esters, preserving desired aromas. Regarding the compounds formed by metabolism of $S$. cerevisiae and $D$. bruxellensis, several previously identified compounds appeared in the fermented beers. For instance, isobutyl acetate appeared in beers 

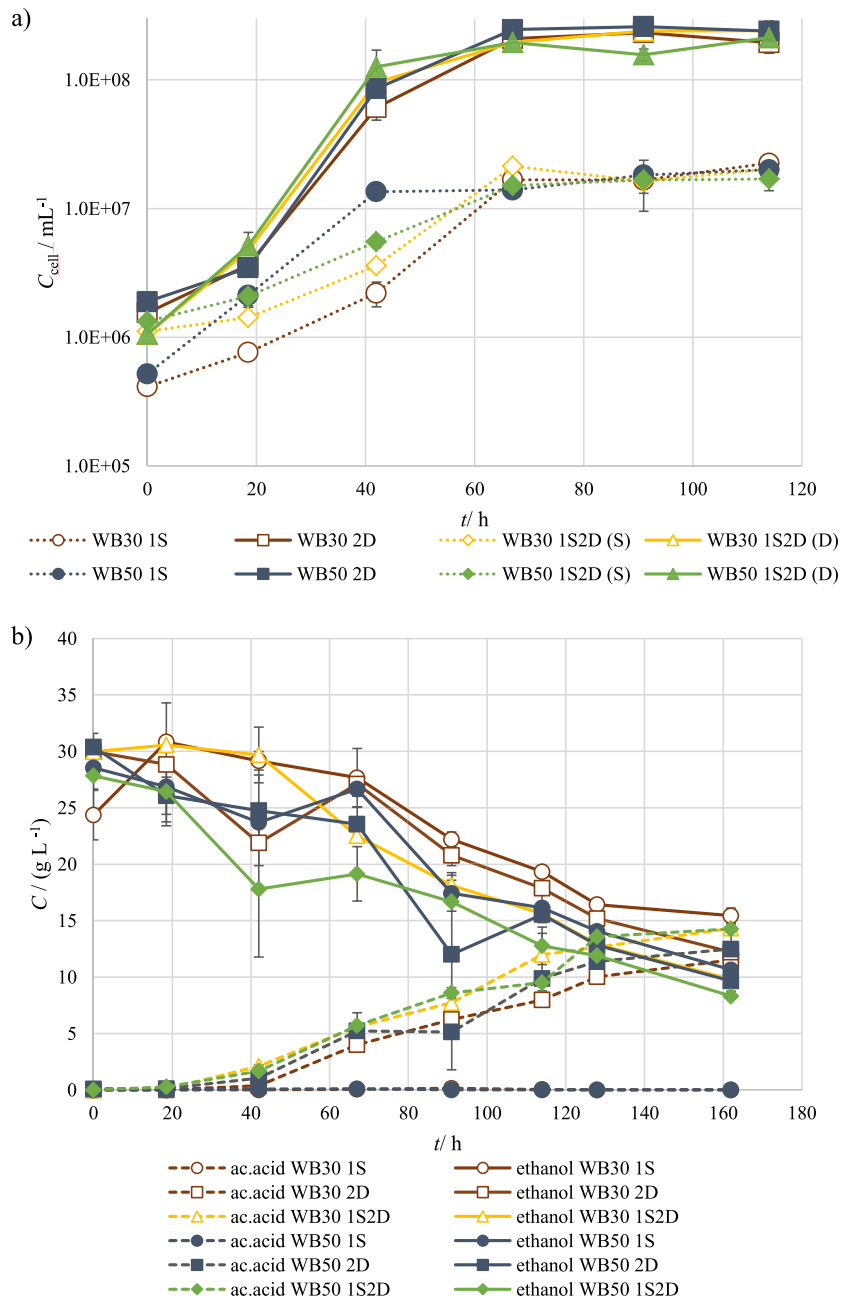

Fig. 5. a) Cell concentration evolution $\left(C_{\text {cell }}\right)$ of $S$. cerevisiae (S) and D. bruxellensis (D) and b) primary metabolite concentration $(C)$ throughout time $(t)$, in fermentations of accelerated aged beer put in contact with wood at $30^{\circ} \mathrm{C}$ (WB30) and $50{ }^{\circ} \mathrm{C}$ (WB50), using pure cultures (2S and 2D) and co-cultures favoring $S$. cerevisiae (2SD) or D. bruxellensis (S2D). $x$ SyD stands for $S$. cerevisiae cellular concentration of $x \times 10^{6} \mathrm{~mL}^{-1}$ and $y \mathrm{D}$ stands for $D$. bruxellensis cellular concentration of $y \times 10^{6} \mathrm{~mL}^{-1}$ in the starter inoculum. Error bars depict standard error of independent duplicates.

fermented with $D$. bruxellensis and in higher concentrations in the coculture. Again, several ethyl and acetate esters were formed mainly by D. bruxellensis, which contribute to the enhancement of fruity aroma in the accelerated aged beers. Also, production of volatile acids was more pronounced in cultures with $D$. bruxellensis, occurring at similar levels either in pure or co-cultures. Similar concentrations were also observed for the production of $D$. bruxellensis characteristic volatile phenols, 4ethylphenol and 4-ethylguayacol either in pure or co-cultures of D. bruxellensis with S. cerevisiae. Also, concentration of ethylphenols was within the same range for the two tested beers implying that extraction of ethylphenol precursors was similar in both conditions using either $30^{\circ} \mathrm{C}$ or $50{ }^{\circ} \mathrm{C}$. On a broader analysis, production of D. bruxellensis key secondary metabolites was similar, either in pure or in coculture of accelerated aged beers. This reinforces that in low sugar concentrations and in the presence of ethanol, the competition between the two yeasts for accessing to the substrate is modified. With ethanol as the main and abundant carbon source, $D$. bruxellensis is able to compete with $S$. cerevisiae, being its traits more easily displayed in the end-product. Scatterplots presented in Fig. 3c) demonstrate clearly this mechanism, with co-culture fermentations appearing closely to D. bruxellensis pure culture fermentations. Also, fermentations including
D. bruxellensis appeared closely with regard to the first component, which distinguishes them from $S$. cerevisiae pure culture fermentations, whereas the second extracted component distinguishes beers prepared with wood at $30^{\circ} \mathrm{C}$ from beers prepared at $50^{\circ} \mathrm{C}$.

\section{Conclusions}

Both Saccharomyces cerevisiae and Dekkera bruxellensis are able to grow simultaneously in co-culture conditions. Medium composition strongly impacts the competition between S.cerevisiae and $D$. bruxellensis, which can be further influenced by pitching concentrations of each species depending on the medium. The differences in growth and competition are reflected in the metabolic profiles obtained in the end-product, being the use of co-cultures an effective strategy to amplify or minimize the production of target species metabolites. Ethanol and wood extract composition are inhibitory of $S$. cerevisiae and D. bruxellensis metabolism and production of D. bruxellensis related metabolites is favored over $S$. cerevisiae metabolites in low glucose/high ethanol conditions. Fermentation using multi-starter S. cerevisiae/ $D$. bruxellensis cultures is a useful tool for the accelerated biological ageing of beer with targeted sensory profiles and furthermore suitable for integration with other accelerated ageing strategies. Therefore, the established hypothesis is confirmed.

\section{CRediT authorship contribution statement}

Eduardo Coelho: Conceptualization, Methodology, Formal analysis, Investigation, Writing - original draft, Writing - review \& editing, Visualization. Mário Azevedo: Investigation. José A. Teixeira: Resources, Writing - review \& editing, Project administration, Funding acquisition. Teresa Tavares: Writing - review \& editing, Supervision, Project administration, Funding acquisition. José M. Oliveira: Conceptualization, Resources, Writing - review \& editing, Supervision, Project administration. Lucília Domingues: Conceptualization, Methodology, Resources, Writing - review \& editing, Supervision, Project administration, Funding acquisition.

\section{Declaration of Competing Interest}

The authors declare that they have no known competing financial interests or personal relationships that could have appeared to influence the work reported in this paper.

\section{Acknowledgements}

This study was supported by the Portuguese Foundation for Science and Technology(FCT) under the scope of the strategic funding of UIDB/ 04469/2020 unit and BioTecNorte operation (NORTE-01-0145-FEDER000004) funded by the European Regional Development Fund under the scope of Norte2020 - Programa Operacional Regional do Norte. Fermentum - Engenharia das Fermentações Lda. also participated in cofunding this work, which authors would like to acknowledge. Lastly, authors would like to thank Mr. Benoît Verdier and Seguin Moreau for supplying the woods and Mr. Paulo Coutinho and Quinta do Portal for supplying the fortified wine.

\section{References}

Abbott, D. A., Hynes, S. H., \& Ingledew, W. M. (2005). Growth rates of Dekkera/ Brettanomyces yeasts hinder their ability to compete with Saccharomyces cerevisiae in batch corn mash fermentations. Applied Microbiology and Biotechnology, 66(6), 641-647. https://doi.org/10.1007/s00253-004-1769-1.

Aguilar-Uscanga, M. G., Garcia-Alvarado, Y., Gomez-Rodriguez, J., Phister, T., Delia, M. L., \& Strehaiano, P. (2011). Modelling the growth and ethanol production of Brettanomyces bruxellensis at different glucose concentrations. Letters in Applied Microbiology, 53(2), 141-149. https://doi.org/10.1111/j.1472-765X.2011.03081.x. Bassi, A. P. G., Silva, J. C. G., Reis, V. R., \& Ceccato-Antonini, S. R. (2013). Effects of 
single and combined cell treatments based on low $\mathrm{pH}$ and high concentrations of ethanol on the growth and fermentation of Dekkera bruxellensis and Saccharomyces cerevisiae. World Journal of Microbiology and Biotechnology, 29(9), 1661-1676. https://doi.org/10.1007/s11274-013-1329-x.

Blomqvist, J., South, E., Tiukova, L., Momeni, M. H., Hansson, H., Ståhlberg, J., .. Passoth, V. (2011). Fermentation of lignocellulosic hydrolysate by the alternative industrial ethanol yeast Dekkera bruxellensis. Letters in Applied Microbiology, 53(1), 73-78. https://doi.org/10.1111/j.1472-765X.2011.03067.x.

Yu, A. Q., Pratomo Juwono, N. K., Foo, J. L., Leong, S. S. J., \& Chang, M. W. (2016). Metabolic engineering of Saccharomyces cerevisiae for the overproduction of short branched-chain fatty acids. Metabolic Engineering, 34, 36-43. https://doi.org/10. 1016/j.ymben.2015.12.005.

Blomqvist, Johanna. (2011). Dekkera bruxellensis-a competitive yeast for ethanol production from conventional and non-conventional substrates. In Sciences-New York. Retrieved from https://pub.epsilon.slu.se/8404/.

Blomqvist, J., Eberhard, T., Schnürer, J., \& Passoth, V. (2010). Fermentation characteristics of Dekkera bruxellensis strains. Applied Microbiology and Biotechnology, 87(4), 1487-1497. https://doi.org/10.1007/s00253-010-2619-y.

Carrau, F. M., Medina, K., Boido, E., Farina, L., Gaggero, C., Dellacassa, E., ... Henschke, P. A. (2005). De novo synthesis of monoterpenes by Saccharomyces cerevisiae wine yeasts. FEMS Microbiology Letters, 243(1), 107-115. https://doi.org/10.1016/j. femsle.2004.11.050.

Ciani, M., \& Comitini, F. (2015). Yeast interactions in multi-starter wine fermentation. Current Opinion in Food Science, 1, 1-6. https://doi.org/10.1016/j.cofs.2014.07.001.

Coelho, E., Domingues, L., Teixeira, J. A., Oliveira, J. M., \& Tavares, T. (2019). Understanding wine sorption by oak wood: Modeling of wine uptake and characterization of volatile compounds retention. Food Research International, 116, 249-257. https://doi.org/10.1016/j.foodres.2018.08.025.

Coelho, E., Lemos, M., Genisheva, Z., Domingues, L., Vilanova, M., \& Oliveira, J. M. (2020). Validation of a LLME/GC-MS methodology for quantification of volatile compounds in fermented beverages. Molecules, 25(3), 1-10. https://doi.org/10. 3390/molecules25030621.

Coelho, E., Magalhães, J., Pereira, F. B., Macieira, F., Domingues, L., \& Oliveira, J. M. (2019). Volatile fingerprinting differentiates diverse-aged craft beers. LWT-Food Science and Technology, 108, 129-136. https://doi.org/10.1016/j.lwt.2019.03.044.

Coelho, E., Teixeira, J. A., Domingues, L., Tavares, T., \& Oliveira, J. M. (2019). Factors affecting extraction of adsorbed wine volatile compounds and wood extractives from used oak wood. Food Chemistry, 295, 156-164. https://doi.org/10.1016/j.foodchem. 2019.05.093.

Colomer, M. S., Funch, B., \& Forster, J. (2019). The raise of Brettanomyces yeast species for beer production. Current Opinion in Biotechnology, 56, 30-35. https://doi.org/10. 1016/j.copbio.2018.07.009.

Comitini, F., Gobbi, M., Domizio, P., Romani, C., Lencioni, L., Mannazzu, I., \& Ciani, M. (2011). Selected non-Saccharomyces wine yeasts in controlled multistarter fermentations with Saccharomyces cerevisiae. Food Microbiology, 28(5), 873-882. https://doi. org $/ 10.1016 / \mathrm{j} . \mathrm{fm} .2010 .12 .001$.

Cunha, J. T., Romaní, A., Costa, C. E., Sá-Correia, I., \& Domingues, L. (2019). Molecular and physiological basis of Saccharomyces cerevisiae tolerance to adverse lignocellulose-based process conditions. Applied Microbiology and Biotechnology, 103(1), 159-175. https://doi.org/10.1007/s00253-018-9478-3.

Denby, C. M., Li, R. A., Vu, V. T., Costello, Z., Lin, W., Chan, L. J. G., ... Keasling, J. D. (2018). Industrial brewing yeast engineered for the production of primary flavor determinants in hopped beer. Nature Communications, 9(1), 1-10. https://doi.org/10. 1038/s41467-018-03293-x.

Galafassi, S., Merico, A., Pizza, F., Hellborg, L., Molinari, F., Piškur, J., \& Compagno, C. (2011). Dekkera/Brettanomyces yeasts for ethanol production from renewable sources under oxygen-limited and low-pH conditions. Journal of Industrial Microbiology and Biotechnology, 38(8), 1079-1088. https://doi.org/10.1007/s10295-010-0885-4.

Hazelwood, L. A., Daran, J. M., Van Maris, A. J. A., Pronk, J. T., \& Dickinson, J. R. (2008). The Ehrlich pathway for fusel alcohol production: A century of research on Saccharomyces cerevisiae metabolism. Applied and Environmental Microbiology, 74(8), 2259-2266. https://doi.org/10.1128/AEM.02625-07.

Holt, S., Mukherjee, V., Lievens, B., Verstrepen, K. J., \& Thevelein, J. M. (2018). Bioflavoring by non-conventional yeasts in sequential beer fermentations. Food Microbiology, 72, 55-66. https://doi.org/10.1016/j.fm.2017.11.008.

Joseph, C. M. L., Albino, E. A., Ebeler, S. E., \& Bisson, L. F. (2015). Brettanomyces bruxellensis aroma-active compounds determined by SPME GC-MS olfactory analysis. American Journal of Enology and Viticulture, 66(3), 379-387. https://doi.org/10. 5344/ajev.2015.14073.

Joseph, C. M. L., Gorton, L. W., Ebeler, S. E., \& Bisson, L. F. (2013). Production of volatile compounds by wine strains of Brettanomyces bruxellensis grown in the presence of different precursor substrates. American Journal of Enology and Viticulture, 64(2), 231-240. https://doi.org/10.5344/ajev.2013.12095.

Kelbert, M., Romaní, A., Coelho, E., Pereira, F. B., Teixeira, J. A., \& Domingues, L. (2016). Simultaneous saccharification and fermentation of hydrothermal pretreated lignocellulosic biomass: Evaluation of process performance under multiple stress conditions. Bioenergy Research, 9(3), https://doi.org/10.1007/s12155-016-9722-6.

Kuo, H. P., Wang, R., Huang, C. Y., Lai, J. T., Lo, Y. C., \& Huang, S. T. (2018). Characterization of an extracellular $\beta$-glucosidase from Dekkera bruxellensis for resveratrol production. Journal of Food and Drug Analysis, 26(1), 163-171. https://doi. org/10.1016/j.jfda.2016.12.016.

Osburn, K., Amaral, J., Metcalf, S. R., Nickens, D. M., Rogers, C. M., Sausen, C., ... Bochman, M. L. (2018). Primary souring: A novel bacteria-free method for sour beer production. Food Microbiology, 70, 76-84. https://doi.org/10.1016/j.fm.2017.09. 007.

Piškur, J., Ling, Z., Marcet-Houben, M., Ishchuk, O. P., Aerts, A., LaButti, K., ... Phister, T. (2012). The genome of wine yeast Dekkera bruxellensis provides a tool to explore its food-related properties. International Journal of Food Microbiology, 157(2), 202-209. https://doi.org/10.1016/j.ijfoodmicro.2012.05.008.

Rodrigues, N., Gonçalves, G., Pereira-da-Silva, S., Malfeito-Ferreira, M., \& Loureiro, V. (2001). Development and use of a new medium to detect yeasts of the genera Dekkera/Brettanomyces. Journal of Applied Microbiology, 90(4), 588-599. https://doi. org/10.1046/j.1365-2672.2001.01275.x.

Rossouw, D., \& Bauer, F. F. (2016). Exploring the phenotypic space of non-Saccharomyces wine yeast biodiversity. Food Microbiology, 55, 32-46. https://doi.org/10.1016/j.fm. 2015.11.017.

Rozpedowska, E., Hellborg, L., Ishchuk, O. P., Orhan, F., Galafassi, S., Merico, A., ... Piškur, J. (2011). Parallel evolution of the make-accumulate-consume strategy in Saccharomyces and Dekkera yeasts. Nature Communications, 2(1), https://doi.org/10 1038/ncomms1305.

Schifferdecker, A. J., Dashko, S., Ishchuk, O. P., \& Piškur, J. (2014). The wine and beer yeast Dekkera bruxellensis. Yeast, 31(9), 323-332. https://doi.org/10.1002/yea.3023.

Smith, B. D., \& Divol, B. (2016). Brettanomyces bruxellensis, a survivalist prepared for the wine apocalypse and other beverages. Food Microbiology, 59, 161-175. https://doi. $\operatorname{org} / 10.1016 / j . f m .2016 .06 .008$.

Smith, B. D., \& Divol, B. (2018). The carbon consumption pattern of the spoilage yeast Brettanomyces bruxellensis in synthetic wine-like medium. Food Microbiology, 73 39-48. https://doi.org/10.1016/j.fm.2017.12.011.

Snauwaert, I., Roels, S. P., Van Nieuwerburg, F., Van Landschoot, A., De Vuyst, L., \& Vandamme, P. (2016). Microbial diversity and metabolite composition of Belgian red-brown acidic ales. International Journal of Food Microbiology, 221, 1-11. https:// doi.org/10.1016/j.ijfoodmicro.2015.12.009.

Spaepen, M., \& Verachtert, H. (1982). Esterase activity in the genus Brettanomyces. Journal of the Institute of Brewing, 88(1), 11-17. https://doi.org/10.1002/j.2050 0416.1982.tb04061.x.

Spitaels, F., Wieme, A. D., Janssens, M., Aerts, M., Daniel, H. M., Van Landschoot, A., ... Vandamme, P. (2014). The microbial diversity of traditional spontaneously fermented lambic beer. PLoS ONE, 9(4), https://doi.org/10.1371/journal.pone. 0095384.

Steensels, J., Daenen, L., Malcorps, P., Derdelinckx, G., Verachtert, H., \& Verstrepen, K. J. (2015). Brettanomyces yeasts - From spoilage organisms to valuable contributors to industrial fermentations. International Journal of Food Microbiology, 206, 24-38. https://doi.org/10.1016/j.ijfoodmicro.2015.04.005.

Suárez, R., Suárez-Lepe, J. A., Morata, A., \& Calderón, F. (2007). The production of ethylphenols in wine by yeasts of the genera Brettanomyces and Dekkera: A review. Food Chemistry, 102(1), 10-21. https://doi.org/10.1016/j.foodchem.2006.03.030.

Sumby, K. M., Grbin, P. R., \& Jiranek, V. (2010). Microbial modulation of aromatic esters in wine: Current knowledge and future prospects. Food Chemistry, 121(1), 1-16. https://doi.org/10.1016/j.foodchem.2009.12.004

Tashiro, Y., Desai, S. H., \& Atsumi, S. (2015). Two-dimensional isobutyl acetate production pathways to improve carbon yield. Nature Communications, 6(May), 1-9. https://doi.org/10.1038/ncomms8488. 